\title{
A multifunctional small RNA binding protein for sensing and signaling cell envelope precursor availability in bacteria
}

\author{
Muna A. Khan ${ }^{1}$ and Boris Görke ${ }^{1, *}$ \\ ${ }^{1}$ Department of Microbiology, Immunobiology and Genetics, Max Perutz Labs, University of Vienna, Vienna Biocenter (VBC), 1030 \\ Vienna, Austria. \\ * Corresponding Author: \\ Boris Görke, Department of Microbiology, Immunobiology and Genetics, Max Perutz Labs, University of Vienna, Vienna Biocenter \\ (VBC), 1030 Vienna, Austria; E-mail: boris.goerke@univie.ac.at
}

Synthesis of glucosamine-6-phosphate (GIcN6P) by the enzyme GImS initiates bacterial cell envelope biosynthesis. To ensure ongoing synthesis, GlcN6P homeostasis is required. Escherichia coli achieves this through a post-transcriptional control mechanism comprising the RNA-binding protein RapZ and small RNAs (sRNAs) GImY and GImZ. GImZ stimulates glmS translation by base-pairing. When GlcN6P is abundant, GImZ is cleaved and inactivated by endoribonuclease RNase $E$. Cleavage depends on RapZ, which binds GImZ and recruits RNase E. Decreasing GIcN6P concentrations provoke up-regulation of the decoy SRNA GImY which sequesters RapZ, thereby suppressing GImZ decay. In our current study we identify RapZ as the GIcN6P sensor. GlcN6P-free RapZ interacts with and stimulates phosphorylation of the two-component system (TCS) QseE/QseF triggering glm $Y$ expression. Thereby generated GImY sequesters RapZ into stable complexes, allowing for glmS expression. Sequestration by GImY also disables RapZ to stimulate QseE/QseF, providing a negative feed-back loop limiting the response. When GIcN6P is replenished, GImY is released from RapZ and rapidly degraded. Our work has revealed a complex regulatory scenario, in which an RNA binding protein senses a metabolite and communicates with two SRNAs, a TCS and ribonuclease RNase E to achieve metabolite homeostasis.

In recent years, post-transcriptional regulation exerted by sRNAs and RNA-binding proteins (RBPs) emerged as crucial layer for regulation of cellular activities in all organisms.
However, only a handful of such RBPs have been characterized in bacteria, leaving important questions in the field unsolved. Traditionally, it is thought that RBPs control RNA fates but how they themselves are controlled and whether this could also involve RNA is largely unclear. It is also unknown how and to what extent RBPs are embedded in the protein-protein interaction network to possibly form higher-order complexes and whether there is direct cross-talk with transcriptional regulators.

In our recent study, we addressed these questions for the RBP RapZ in E. coli. Previous work has shown that RapZ acts in concert with two homologous sRNAs GImY and GImZ to achieve homeostasis of the key metabolite GlcN6P. All amino sugar constituents of the bacterial cell envelope, namely peptidoglycan and outer membrane lipopolysaccharides, derive from GlcN6P. The need for GlcN6P synthesis may change during the life-cycle of a bacterium depending on growth rate, extracellular amino sugar levels and presence of Glms inhibitory antimicrobials produced by competing microorganisms. Therefore, bacteria must sense intracellular GlcN6P and adjust GImS activity accordingly. To this end, many species rely on post-transcriptional mechanisms. In E. coli, the ribosomal binding site of the glmS mRNA is masked by an inhibitory stem loop structure. Assisted by the RNA chaperone Hfq, sRNA GImZ base-pairs with the gImS mRNA and resolves this structure. Consequently, translation occurs, which concomitantly protects the mRNA from degradation (Fig. 1). GImZ itself is controlled at the level of decay. The endoribonuclease RNase $\mathrm{E}$ inactivates $\mathrm{GlmZ}$ by cleaving within its base-pairing site (Fig. 1, left). However, RNase E cannot act alone on GImZ. Processing requires RapZ - the $\underline{\mathbf{R} N a s e} \mathrm{E}$ adaptor protein for

MICROREVIEW on: Khan MA, Durica-Mitic S, Göpel Y, Heermann R, Görke B (2020). Small RNA-binding protein RapZ mediates cell envelope precursor sensing and signaling in Escherichia coli. EMBO J 39(6):e103848. doi: 10.15252/embj.2019103848 


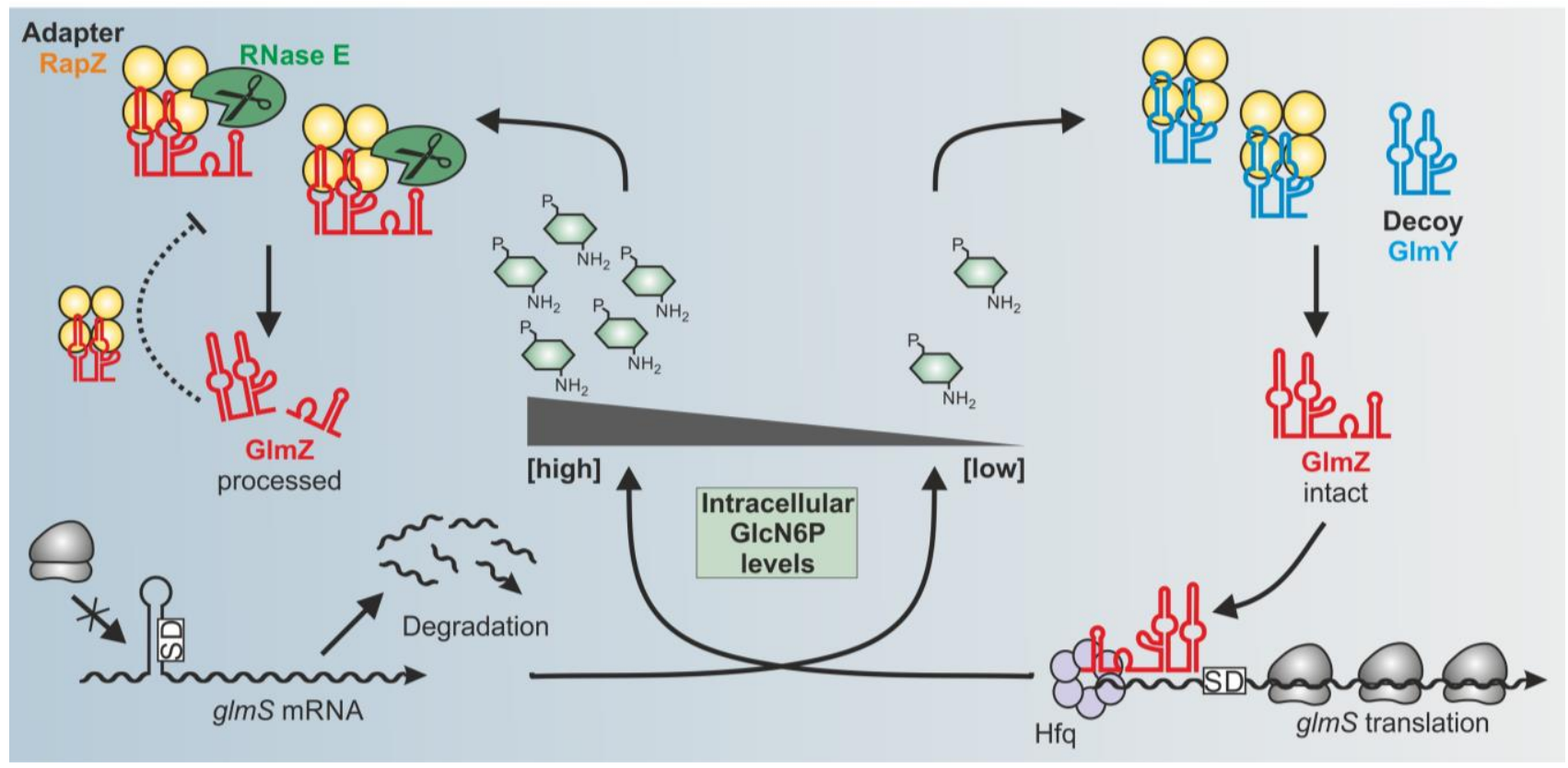

FIGURE 1: Model for the control of glmS mRNA translation by the GImY/RapZ/GImZ circuit in response to GlcN6P sufficiency (left) and starvation (right) conditions.

cleavage of GImZ. RapZ interacts with RNase E and also binds GImZ at its central stem loop using a C-terminal noncanonical RNA binding domain enriched in positively charged residues. RapZ, a tetramer, is envisioned to form an encounter complex with tetrameric RNase $E$ to cleave the sandwiched SRNA. Under GIcN6P sufficiency, the majority of GImZ molecules undergoes cleavage by the RapZ/RNase E complex, resulting in basal GImS levels (Fig. 1, left). This basal level is adjusted by an inhibitory feedback loop mediated by the processed GImZ variant, which retains the ability to bind RapZ. When processed GImZ accumulates, it sequesters RapZ at least partially, reducing ongoing GlmZ processing (Fig. 1, left). Upon GlcN6P scarcity, a second sRNA enters the game: GImY is homologous to GImZ, but lacks the $g / m S$ base-pairing site. Moreover, GImY has low affinity for $\mathrm{Hfq}$ as $\mathrm{Hfq}$-binding motifs are lacking or sequestered by secondary structure. Accordingly, GImY does not act via base-pairing but through sequestration of protein RapZ. A processed variant of GImY, generated by a yet unknown activity, specifically accumulates when GIcN6P concentrations decrease and sequesters RapZ through molecular mimicry (Fig. 1, right). Consequently, GImZ stays intact leading to increased GImS amounts that replenish GlcN6P. Experimentally, this cascade can be induced by antibiotics targeting GlmS, providing a tool for analysis. For instance, Nva-FMDP - a derivative of a dipeptide produced by Streptomyces collinus - binds and inhibits GImS, blocking GlcN6P production. As a result, GImY accumulates and increases GImS synthesis through GImZ. Interestingly, higher GlmS levels overcome inhibition by the drug, providing intrinsic resistance. Thus, the need for defense against antibiotics released by competing microorganisms may provide one explanation why many bacteria including $E$. coli feedback-regulate GImS at the level of enzyme synthesis and not activity as observed in eukaryotes.

Until recently, it remained mysterious how the GIcN6P signal is sensed and processed by the GlmY/RapZ/GImZ circuit. As $g / m Y$ mutants fail to activate $g / m S$ expression under GIcN6P depletion, we initially hypothesized that GImY itself or a factor upstream might sense this metabolite. Contrarily, our recent study revealed that in fact RapZ represents the sought GIcN6P sensor. RapZ binds GIcN6P in vivo as shown by targeted metabolomics, in which GIcN6P co-eluted with RapZ purified by affinity chromatography. Surface plasmon resonance spectroscopy (SPR) demonstrated specific and high affinity binding in vitro. Decreasing GlcN6P concentrations trigger incremental glmY transcription rates and rapZ mutants fail to do so. These findings indicated that RapZ stimulates $g \operatorname{lm} Y$ expression to sequester itself when sensing a low GlcN6P level. How can an RBP activate transcription initiation? Transcription of $g / m Y$ can be initiated from two overlapping $\sigma^{54}$ and $\sigma^{70}$ promoters. Whereas the latter is weak and unregulated, the stronger $\sigma^{54}$ promoter is controlled by the TCS QseE/QseF. This system consists of a cytoplasmic membrane bound kinase (QseE) that auto-phosphorylates and subsequently transfers the phosphoryl-group to the response regulator (QseF), which then binds upstream of the glm $Y$ promoter to activate transcription initiation (Fig. 2A). Notably, we found that RapZ activates QseE/QseF from inside the cell. Two-hybrid assays and SPR indicate that RapZ binds QseE as well as QseF. Concomitantly, RapZ increases auto-phosphorylation of QseE and thereby phospho-transfer to QseF, as revealed by genetic analyses and in vitro phosphorylation assays. GlcN6P-bound RapZ is unable to stimulate QseE/QseF. Thus, dependent on metabo- 


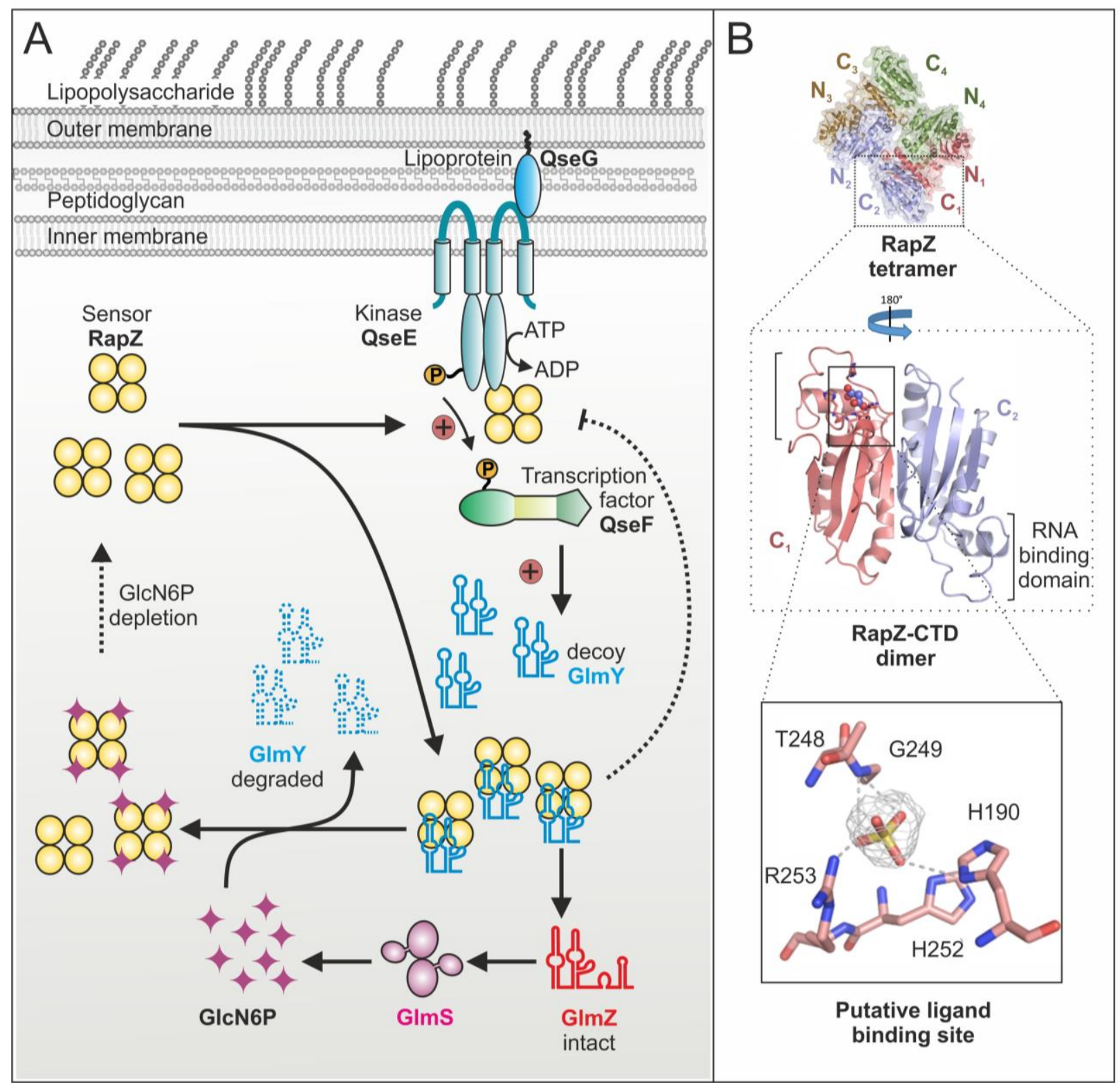

FIGURE 2: GIcN6P sensing and signaling by RapZ. (A) Regulatory cascade initiated by RapZ when sensing GlcN6P scarcity. (B) Cartoon representation of the X-ray crystal structure of tetrameric RapZ (upper panel). The middle panel shows localization of the RNA binding domain and a putative ligand binding pocket occupied by malonate within the RapZ-CTD swapped dimer. Bottom panel: Close-up view of the residues forming the presumptive GICN6P binding site with bound sulfate. The cartoons illustrating the RapZ structure are from Gonzalez et al., 2017, Nucleic Acids Res. 45(18):10845-10860.

lite availability Rapz engages in a higher order protein complex to transfer information to the transcription factor controlling its decoy sRNA. The resulting higher GImY levels sequester RapZ into stable complexes, titrating it away from GImZ, which then activates glmS expression (Fig. 2A). This regulatory mechanism also includes a novel type of feedback loop: GImY binding interferes with the ability of RapZ to activate QseE/QseF (Fig. 2A). Thus, a sRNA abrogates communication between two signaling proteins. Thereby, $g / m Y$ expression is fine-tuned to the level required to saturate all available RapZ molecules, limiting the response.
Importantly, GIcN6P not only controls activation of QseE/QseF by RapZ but also governs interaction of RapZ with sRNA GImY: Once replenished, GIcN6P releases GImY from RapZ leading to rapid degradation of the sRNA as revealed by EMSA and GImY half-life determinations (Fig. 2A). Consequently, the accumulation of GImY observed under GlcN6P starvation conditions results from two distinct activities of RapZ: Up-regulation of $g / m Y$ expression and protection of the produced GImY through binding. Taken together, our work reveals a multifunctional bacterial sRNA-binding protein whose activity is directly controlled by a metabolite. When GlcN6P is present, RapZ 
primarily acts as adaptor protein for cleavage of GImZ by RNase E. When GlcN6P is absent, RapZ communicates with a signaling complex to increase expression of its titrating decoy GlmY. Thus, RapZ regulates and is regulated by one and the same sRNA.

What are the interaction sites used by RapZ to contact its various partners? According to the crystal structure, the RapZ monomer consists of well-separated N-terminal and C-terminal globular domains (NTD/CTD). Four monomers form a domain-swapped dimer of dimers held together through three types of inter-protomer contacts (Fig. 2B, top). The tetrameric assembly of RapZ is essential for binding RNase E as well as QseE/QseF and initial data suggest that both domains of RapZ contribute to these interactions. The RapZ-CTD can form a swapped dimer independently, which may bind sRNA GImY and GICN6P in a mutually exclusive manner (Fig. 2B, middle panel). Intriguingly, the CTD contains a pocket that could accommodate the phosphate moiety of a non-protein ligand and is located in close proximity to the RNA-binding domain (Fig. 2B, middle and bottom panel). Thus, it might be speculated that GlcN6P and GImY compete for binding to the RapZ-CTD. As interaction of RapZ with QseE/QseF is counteracted by GlcN6P as well as SRNA binding, this region may also be part of the surface contacting the TCS. In contrast, the role of the RapZ-NTD is less obvious. The separated RapZ-CTD has higher affinity towards both GIcN6P and GImY than fulllength RapZ. Thus, the NTD might have an auto-inhibitory role. Whether this may be regulated by a different metabolite, as suggested by occurrence of a nucleotide-binding motif in the NTD, remains to be clarified.

Whereas the response of the GImY/RapZ/GImZ circuit to GIcN6P starvation is now well understood, it remains unclear how GIcN6P interferes with binding and cleavage of GImZ by the RapZ/RNase E complex. Intuitively, binding of GIcN6P is expected to stimulate RapZ-mediated decay of GImZ, but so far there is no evidence that RapZ could discriminate between the two sRNAs. On the other hand, our data indicate that even under normal (i.e. GIcN6P sufficiency) conditions, a fraction of the RapZ molecules remain GIcN6P-free. Interestingly, the putative binding pocket in the RapZ-CTD (Fig. 2B) bears homology to the phosphatase loop found in cysteine-dependent protein-tyrosine phosphatases. Perhaps RapZ disengages itself from GIcN6P over time through hydrolysis, allowing it to trigger GImZ decay when GIcN6P is replenished - a hypothesis that is currently under investigation.

We previously demonstrated that QseE/QseF relies on a third component for function: using its periplasmic domains, QseE interacts with the outer-membrane attached lipoprotein QseG to gain kinase activity (Fig. 2A). Thus, for a fully activated TCS, QseE must be contacted by QseG from outside and by RapZ from inside, forming a large trans-envelope signaling complex. Through integration of RapZ, the QseE/QseF TCS is recruited to the GIcN6P starvation signal. Interestingly, QseF also activates expression of $r p o E$ encoding the sigma factor orchestrating the cell envelope stress response. It is possible that QseG senses a process in the envelope - perhaps integrity of an amino sugar containing component - to adjust synthesis of cell envelope precursors and envelope repair mechanisms accordingly.

In $E$. coli $\mathrm{K}-12 \mathrm{~g} / \mathrm{mS}$ is the only known target gene regulated by GImY/GImZ. In EHEC these sRNAs were recruited to regulate virulence genes, and mutations in QseEGF strongly interfere with virulence in EHEC and other enterobacterial pathogens. Possibly, these pathogens coordinate virulence gene expression with changes in cell envelope synthesis or integrity that may be encountered in the host environment and sensed by QseEGF. In this respect, the GlmY/RapZ/GImZ circuit and the QseEGF machinery could provide attractive targets for antimicrobial chemotherapy. We previously demonstrated that co-application of the non-metabolizable GlcN6P analog glucosamine-6-sulfate $\left(\mathrm{GlcN6SO}_{4}\right)$ increases the efficacy of GlmS inhibitors such as Nva-FMDP. GlcN6SO 4 is only weakly active, but understanding how GIcN6P interacts with RapZ may provide a rational basis for designing more potent compounds suppressing GImY/RapZ/GImZ. Application of such compounds might not only render cells more susceptible to antibiotics targeting GlmS, but is also expected to suppress virulence of pathogenic Enterobacteriaceae.

\section{ACKNOWLEDGMENTS}

We thank all authors who contributed to the original study discussed in this review. The original work was supported by stand-alone grants P26681 and P32410 of the "Austrian Science Fund" (FWF) to B.G. and the Doktoratskolleg RNA Biology W1207-B09.

\section{CONFLICT OF INTEREST}

The authors declare that they have no conflict of interest.

\section{COPYRIGHT}

(C) 2020 Khan and Görke. This is an open-access article released under the terms of the Creative Commons Attribution (CC BY) license, which allows the unrestricted use, distribution, and reproduction in any medium, provided the original author and source are acknowledged.

Please cite this article as: Muna A. Khan and Boris Görke (2020). A multifunctional small RNA binding protein for sensing and signaling cell envelope precursor availability in bacteria. Microbial Cell 7(5): 139-142. doi: 10.15698/mic2020.05.717 